\title{
Relaxing Goodness is Still Good
}

\author{
Gordon J. Pace ${ }^{1}$ and Gerardo Schneider ${ }^{2}$ \\ 1 Dept. of Computer Science and AI, University of Malta, Msida, Malta. \\ 2 Dept. of Informatics, University of Oslo, Oslo, Norway. \\ \{gordon.pace@um.edu.mt; gerardo@ifi.uio.no\}
}

\begin{abstract}
Polygonal hybrid systems (SPDIs) are planar hybrid systems, whose dynamics are defined in terms of constant differential inclusions, one for each of a number of polygonal regions partitioning the plane. The reachability problem for SPDIs is known to be decidable, but depends on the goodness assumption which states that the dynamics do not allow a trajectory to both enter and leave a region through the same edge. In this paper we extend the decidability result to generalised SPDIs (GSPDI), SPDIs not satisfying the goodness property, and give an algorithmic solution to decide reachability of such systems.
\end{abstract}

\section{Introduction}

A hybrid system is one in which discrete and continuous behaviours interact. Some systems are inherently hybrid - consider a robot, with differential equations determining its speed, together with an embedded computer taking discrete decisions based on the continuous input values coming from sensors. In other cases, a system consisting only of continuous behaviour, can be hybridised, introducing discrete behaviour in order to facilitate the analysis. For example, exact solutions can be difficult to obtain for a nonlinear differential equation, making a qualitative and approximative analysis necessary. An interesting class of hybrid systems for which the reachability question is known to be decidable, is the class of Polygonal Hybrid Systems (SPDIs) - a subclass of hybrid systems on the plane whose dynamics is defined by constant differential inclusions [ASY01,ASY07]. Informally, an SPDI consists of a partition of the plane into polygonal regions, each of which enforces different dynamics given by two vectors determining the possible directions a trajectory might take; a simple SPDI is depicted in Fig. 1(a). A constructive proof for deciding reachability on SPDIs can be found in [ASY07]. The proof is restricted to SPDIs which have the so-called goodness property - the dynamics of any region of the SPDI do not allow a trajectory to traverse any edge of the polygonal region in opposite directions. An SPDI without the goodness property is called a Generalised SPDI (GSPDI).

Fig. 1-(b) shows an example of a good and a 'bad' region (here 'bad' indicates that the region does not satisfy the goodness criterion). In the figure on the left we can see a good region, where the two vectors $\mathbf{a}$ and $\mathbf{b}$ make it impossible for a trajectory to enter and leave the region $P$ through the same edge of the polygon delimiting the region. On the other hand, the figure on the right shows a bad region: Both $e_{2}$ and $e_{5}$ can be crossed in both directions by a trajectory entering and leaving $P$, as shown in the figure. 


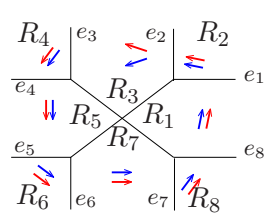

(a)

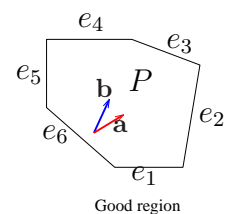

$(b)$

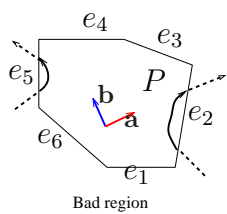

Fig. 1. (a) Example of an SPDI; (b) Good and bad regions.
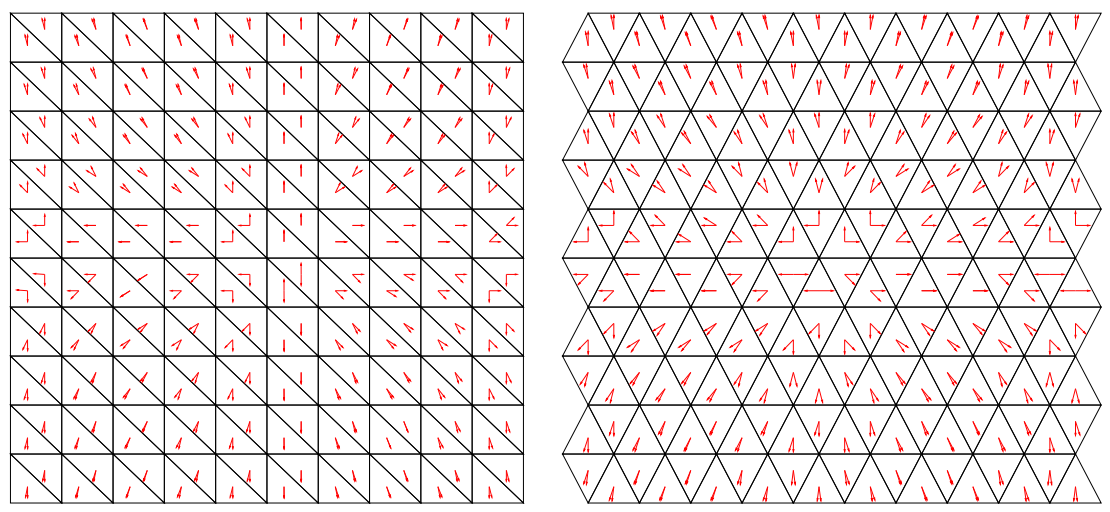

Fig. 2. Approximating a non-linear differential equation using different partitioning of the plane.

The algorithm presented in [ASY07] for deciding reachability on SPDI depends on preprocessing of trajectory segments and a qualitative analysis to guarantee that it is possible to review the behaviour of all the possible signatures ${ }^{1}$, by looking at only a finite set of abstract signatures. Informally, this is achieved as follows: (1) Trajectory segments are simplified - it is sufficient to look at trajectories made up of straight segments across regions, and which do not cross themselves; (2) Trajectory segments are abstracted into signatures, based on the Poincaré map [HS74], that relates $n$-dimensional continuous-time systems with $(n-1)$-dimensional discrete-time systems; (3) It is shown that it is sufficient to look at signatures which consist only of sequences of edges and simple cycles; (4) Such signatures can be abstracted into types of signatures - signatures which do not take into account the number of times each simple cycle is iterated.

Many of the lemmas for proving that the above guarantee the finiteness of types of signatures critically depend on the goodness assumption, which propagate this dependency to the constructive proof given for deciding reachability of SPDIs.

Restricting oneself only to SPDIs satisfying the goodness assumption makes it very difficult to model real-life examples. Unfortunately, extending the SPDI model in most ways, such as allowing jumps with resets (from one edge to another remote one), in-

\footnotetext{
${ }^{1}$ We call signature the sequence of traversed edges by the trajectory. A more formal definition will be given in a later section.
} 
creasing the number of dimensions and allowing non-linear differential inclusions, have been shown to make the model undecidable [AS02].

A potentially interesting and useful application of SPDIs is that of the approximation and analysis of two-dimensional non-linear differential equations. By splitting the plane into polygons, and by setting the dynamics of each polygon to be over-approximations of the non-linear differential equation in that region, one can ask reachability questions about the equation, and obtain answers accordingly. When over-approximating the dynamics, a negative reachability answer implies a negative answer in the exact equation. Using more and smaller polygons enables more precise approximations.

The problem with using this approach is that for most differential equations, using a fixed partition breaks the goodness assumption, since almost invariably, some edges of some regions will lie within the differential inclusion of that region. One solution would be to try to derive an intelligent partition of the plane which maintains goodness, which in some cases may be impossible, or by extending the SPDI analysis algorithms to relax the goodness assumption, thus enabling the modelling of non-linear differential equations in a straightforward manner.

As a simple example, consider a pendulum with friction coefficient $k$, mass $M$, pendulum length $R$ and gravitational constant $g$. If $\theta$ is the angle subtended with the vertical, the behaviour of such a pendulum is described by the differential equation: $M R^{2} \ddot{\theta}+k \dot{\theta}+M g R \sin \theta=0$. By taking $x=\theta$, and $y=\dot{\theta}$, we get $\dot{x}=y$ and

$\dot{y}=-\frac{k y}{M R^{2}}-\frac{g \sin (x)}{R}$. Using these formulae, we can produce SPDIs expressing these constraints, possibly with different plane partitions. Fig. 2 gives two such partitions for $k=1, R=10, M=10$, and $g=-10$. Visual inspection of the SPDIs, shows that various polygons are not good. By presenting an algorithm showing the decidability of reachability on GSPDIs, we can automatically analyse such systems.

In this paper, we present a constructive decidable algorithm for solving the reachability problem for GSPDIs. This decidability result contributes towards narrowing the undecidability frontier of low dimension hybrid systems [AS02,MP05], and it allows GSPDIs to be used to approximate planar non-linear differential equations.

The paper is organised as follows. In the next section we define the notation used, and outline definitions and results about SPDIs. Section 3 is concerned with the extension of these results to enable analysis of GSPDIs, including the decision algorithm for reachability. We conclude in the last section.

\section{Polygonal Hybrid Systems (SPDIs)}

In this section we recall the main definitions and concepts required in the rest of the paper, and give an outline of the results for SPDIs, upon which the results presented in this paper are built. For a more detailed presentation see [ASY07]. In what follows, we will use $\mathbf{a}=\left(a_{1}, a_{2}\right)$ and $\mathbf{x}=\left(x_{1}, x_{2}\right)$ to represent 2-dimensional vectors $\left(\mathbf{a}, \mathbf{x} \in \mathbb{R}^{2}\right)$. An angle $\angle \mathbf{b}$ on the plane, defined by two non-zero vectors $\mathbf{a}$ and $\mathbf{b}$ is the set of all positive linear combinations $\mathbf{x}=\alpha \mathbf{a}+\beta \mathbf{b}$, with $\alpha, \beta \geq 0$, and $\alpha+\beta>0$. We can always assume that $\mathbf{b}$ is situated in the counter-clockwise direction from $\mathbf{a}$.

Definition 1. A polygonal hybrid system (SPDI) is a pair $\mathcal{H}=\langle\mathbb{P}, \mathbb{F}\rangle$, where $\mathbb{P}$ is a finite partition of the plane (with each $P \in \mathbb{P}$ being a convex polygon), called the 
regions of the SPDI, and $\mathbb{F}$ is a function which associates a pair of vectors to each polygon: $\mathbb{F}(P)=\left(\mathbf{a}_{P}, \mathbf{b}_{P}\right)$.

In an SPDI every point on the plane has its dynamics defined according to which polygon it belongs to: if $\mathbf{x} \in P$, then $\dot{\mathbf{x}} \in \angle_{\mathbf{a}_{P}}^{\mathbf{b}_{P}}$.

Example 1. Consider the SPDI illustrated in Fig. 1-(a), with eight regions $R_{1}, R_{2}, \ldots, R_{8}$. A pair of vectors $\left(\mathbf{a}_{i}, \mathbf{b}_{i}\right)$ is also associated to each region $R_{i}: \mathbf{a}_{1}=\mathbf{b}_{1}=(1,5)$, $\mathbf{a}_{2}=\mathbf{b}_{2}=\left(-1, \frac{1}{2}\right), \mathbf{a}_{3}=\left(-1, \frac{11}{60}\right)$ and $\mathbf{b}_{3}=\left(-1,-\frac{1}{4}\right), \mathbf{a}_{4}=\mathbf{b}_{4}=(-1,-1)$, $\mathbf{a}_{5}=\mathbf{b}_{5}=(0,-1), \mathbf{a}_{6}=\mathbf{b}_{6}=(1,-1), \mathbf{a}_{7}=\mathbf{b}_{7}=(1,0), \mathbf{a}_{8}=\mathbf{b}_{8}=(1,1)$.

We define $E(P)$ to be the set of edges of region $P$. We say that an edge $e(e \in E(P))$ is an entry-only of $P$ if for all $\mathbf{x} \in e$ and for all $\mathbf{c} \in \angle_{\mathbf{a}_{P}}^{\mathbf{b}_{P}}, \mathbf{x}+\mathbf{c} \epsilon \in P$ for some $\epsilon>0$. We say that $e$ is an exit-only of $P$ if the same condition holds for some $\epsilon<0$. Intuitively, an entry-only (exit-only) edge of a region $P$ allows at least a trajectory in $P$ starting (terminating) on edge $e$, but allows no trajectories in $P$ terminating (starting) on edge $e$. We write $\operatorname{In}(P)(\operatorname{In}(P) \subseteq E(P))$ to denote the set of all entry-only edges of $P$ and $\operatorname{Out}(P)(\operatorname{Out}(P) \subseteq E(P))$ to denote the set of exit-only edges of $P$. From the definition, it follows immediately that no edge can be both an entry-only and an exit-only edge of a region: $\operatorname{In}(P) \cap \operatorname{Out}(P)=\emptyset$.

A region $P$ is said to be good, if all the edges of that region are either entry-only or exitonly: $E(P)=\operatorname{In}(P) \cup \operatorname{Out}(P)$. An SPDI is said to be good, or satisfy the goodness property, if it consists of only good regions: $\forall P \in \mathbb{P} \cdot E(P)=\operatorname{In}(P) \cup \operatorname{Out}(P)$.

Assumption 1 In the rest of this section, we will consider only good SPDIs.

Example 2. In Fig. 1-(b), the region $P$ shown on the left is good since all edges are either entry-only or exit-only. The region depicted on the right shows a region that is not good, since neither edge $e_{2}$ nor edge $e_{5}$ are in $\operatorname{In}(P) \cup \operatorname{Out}(P)$.

We will use the notation $e_{\circlearrowright}^{P}$ to indicate the directed edge $e$ such that it follows a clockwise direction around region $P$, and similarly $e_{\circlearrowleft}^{P}$ to indicate the directed edge $e$ following an anticlockwise direction around region $P$. Given a directed edge $e$, its inverse will be written as $e^{-1}$.

Definition 2. The set of directed edges of an SPDI $\mathcal{H}$ with partition $\mathbb{P}$, written $E_{d}(\mathcal{H})$, is defined to be: $E_{d}(\mathcal{H})=\left\{e_{\circlearrowright}^{P} \mid P \in \mathbb{P}, e \in \operatorname{In}(P)\right\} \cup\left\{e_{\circlearrowleft}^{P} \mid P \in \mathbb{P}, e \in \operatorname{Out}(P)\right\}$. Similarly, we define $\operatorname{In}_{d}(P)$ and $\operatorname{Out}_{d}(P)$ to correspond to $\operatorname{In}(P)$ and $\operatorname{Out}(P)$ but with directed edges.

Since an edge typically appears in two adjacent regions, the direction induced in the two regions may be different. However, it was proved that edges which are entry-only in one region, and exit-only in the other result in matching induced directions: $e \in E_{d}(\mathcal{H})$ or $e^{-1} \in E_{d}(\mathcal{H})$, but not both [ASY01,MP93]. In an SPDI satisfying goodness, the only case where one can have both $e$ and $e^{-1}$ is when $e$ is an entry-only (or exit-only) edge in both adjacent regions it belongs to.

A trajectory segment of an SPDI $\mathcal{H}$, is a continuous function $\xi \in[0, T] \rightarrow \mathbb{R}^{2}$ such that for all $t \in[0, T]$, if $\xi(t) \in P$ and $\dot{\xi}(t)$ is defined then $\dot{\xi}(t) \in \angle_{\mathbf{a}_{P}}^{\mathbf{b}}$. The signature of a 
trajectory segment $\xi$, written $\operatorname{Sig}(\xi)$, is the ordered sequence of edges traversed by the trajectory, that is, $e_{1}, e_{2}, \ldots e_{n}$ resulting from $\xi \cap E_{d}(\mathcal{H})$.

One of the most important results presented in [ASY07] is that the behaviour of any trajectory is equivalent to the behaviour of some trajectory which does not cross itself and follows straight-line segments within regions.

Lemma 1. Given a trajectory segment $\xi \in[0, T] \rightarrow \mathbb{R}^{2}$, there exists another trajectory segment $\xi^{\prime} \in\left[0, T^{\prime}\right] \rightarrow \mathbb{R}^{2}$ starting and finishing at the same points as $\xi\left(\xi(0)=\xi^{\prime}(0)\right.$ and $\xi(T)=\xi^{\prime}\left(T^{\prime}\right)$ ) such that (i) $\xi^{\prime}$ does not cross itself ( $\xi$ is injective); and (ii) $\xi^{\prime}$ follows straight-line segments inside regions.

This result shows that to decide reachability, it is sufficient to look at non-self-crossing trajectories consisting of straight-line segments. In the rest of the discussion, we will restrict our use of trajectory to mean 'a non-self-crossing trajectory composed of straightline segments between edges'. Similarly, the term signature will be used to indicate the signature of a trajectory with these constraints. Note that the result is true of any SPDI, not only ones satisfying the goodness constraint.

Truncated Affine Multi-Valued Functions. An affine function $f \in \mathbb{R} \rightarrow \mathbb{R}$ is such that $f(x)=a x+b$. If $a>0$ we say that $f$ is positive affine, and if $a<0$ we say that $f$ is negative affine; we call this the parity of the affine function.

An affine multivalued function (AMF) $F \in \mathbb{R} \rightarrow 2^{\mathbb{R}}$, written $F=\left\langle f_{l}, f_{u}\right\rangle$, is defined by $F(x)=\left\langle f_{l}(x), f_{u}(x)\right\rangle$ where $f_{l}$ and $f_{u}$ are affine and $\langle\cdot, \cdot\rangle$ denotes an interval. For notational convenience, we do not make explicit whether intervals are open, closed, left-open or right-open, unless required for comprehension. For an interval $I=\langle l, u\rangle$ we have that $F(\langle l, u\rangle)=\left\langle f_{l}(l), f_{u}(u)\right\rangle$. An inverted affine multivalued function $F \in$ $\mathbb{R} \rightarrow 2^{\mathbb{R}}$, written $F=\left\langle f_{l}, f_{u}\right\rangle$, is defined by $F(x)=\left\langle f_{u}(x), f_{l}(x)\right\rangle$ where $f_{l}$ and $f_{u}$ are both negative affine and $\langle\cdot, \cdot\rangle$ denotes an interval.

Given an AMF $F$ and two intervals $S \subseteq \mathbb{R}^{+}$and $J \subseteq \mathbb{R}^{+}$, a truncated affine multivalued function (TAMF) $\mathcal{F}_{F, S, J} \in \mathbb{R} \rightarrow 2^{\mathbb{R}}$ is defined as follows: $\mathcal{F}_{F, S, J}(x)=F(x) \cap J$ if $x \in S$, otherwise $\mathcal{F}_{F, S, J}(x)=\emptyset$. In what follows we will write $\mathcal{F}$ instead of $\mathcal{F}_{F, S, J}$ whenever no confusion may arise. Moreover, in the rest of the paper $F$ will always denote an AMF and $\mathcal{F}$ a TAMF. For convenience we write $\mathcal{F}(x)=F(\{x\} \cap S) \cap J$ instead of $\mathcal{F}(x)=F(x) \cap J$ if $x \in S$. We overload the application of a TAMF on an interval $I: \mathcal{F}(I)=F(I \cap S) \cap J$. We say that $\mathcal{F}$ is normalised if $S=\operatorname{Dom}(\mathcal{F})=\{x \mid$ $F(x) \cap J \neq \emptyset\}$ and $J=\operatorname{Im}(\mathcal{F})=\mathcal{F}(S)$.

As in the case of affine multivalued functions, an inverted truncated affine multivalued function (inverted TAMF) is similar to a TAMF, but defined in terms of an inverted affine multivalued function as opposed to a normal one. An important result is that normal TAMFs are closed under composition.

Theorem 1. The functional composition of two normal TAMFs $\mathcal{F}_{1}(I)=F_{1}\left(I \cap S_{1}\right) \cap$ $J_{1}$ and $\mathcal{F}_{2}(I)=F_{2}\left(I \cap S_{2}\right) \cap J_{2}$, is the TAMF $\left(\mathcal{F}_{2} \circ \mathcal{F}_{1}\right)(I)=\mathcal{F}(I)=F(I \cap S) \cap J$, where $F=F_{2} \circ F_{1}, S=S_{1} \cap F_{1}^{-1}\left(J_{1} \cap S_{2}\right)$ and $J=J_{2} \cap F_{2}\left(J_{1} \cap S_{2}\right)$.

The following new corollary extends the above result. 
Corollary 1. The composition of two inverted TAMFs gives a normal TAMF. Conversely, the composition of one normal and one inverted TAMF (in either order) gives an inverted TAMF.

To avoid having to reason about the length of every edge, we normalise every edge $e$ such that its TAMF has the domain $[0,1]$ (that is, the normalised version of $e$ has length 1 , with 0 corresponding to the starting point of the directed edge, and 1 to the end point). Successors Given an SPDI, we fix a one-dimensional coordinate system on each edge to represent points lying on edges. For notational convenience, we will use $e$ to denote both the directed edge and its one-dimensional representation. Accordingly, we write $\mathrm{x} \in e$ and $x \in e$, to mean "point $\mathbf{x}$ lies on edge $e$ " and "coordinate $x$ in the onedimensional coordinate system of $e$ " respectively. The same convention applied to sets of points of $e$ represented as intervals (for example, $\mathbf{x} \in I$ and $x \in I$, where $I \subseteq e$ ) and to trajectories (for example, " $\xi$ starting at $x$ " or " $\xi$ starting at $\mathbf{x}$ ").

Consider a polygon $P \in \mathbb{P}$, with $e_{0} \in \operatorname{In}_{d}(P)$ and $e_{1} \in \operatorname{Out}_{d}(P)$. For $I \subseteq e_{0}$, Succ $_{e_{0} e_{1}}(I)$ is defined to be the set of all points lying on $e_{1}$ reachable from some point in $I$ by a trajectory segment $\xi \in[0, t] \rightarrow \mathbb{R}^{2}$ in $P$ (that is, $\xi(0) \in I \wedge \xi(t) \in$ $\left.e_{1} \wedge \operatorname{Sig}(\xi)=e_{0} e_{1}\right)$. Given $I=[l, u]$, $\operatorname{Succ}_{e_{0} e_{1}}(I)=F\left(I \cap S_{e_{0} e_{1}}\right) \cap J_{e_{0} e_{1}}$, where $S_{e_{0} e_{1}}$ and $J_{e_{0} e_{1}}$ are intervals, $F([l, u])=\left\langle f_{l}(l), f_{u}(u)\right\rangle$ and $f_{l}$ and $f_{u}$ are positive affine functions. Successors are thus normal TAMFs.

Qualitative analysis of simple edge-cycles Let $\sigma=\left(e_{1} \ldots e_{k}\right)$ be a simple edge-cycle - that is, a signature that can be repeated a number of times, and such that all edges are distinct $\left(e_{i} \neq e_{j}\right.$ for all $\left.1 \leq i<j \leq k\right)$. Let $\operatorname{Succ}_{\sigma}(I)=F\left(I \cap S_{\sigma}\right) \cap J_{\sigma}$ with $F=\left\langle f_{l}, f_{u}\right\rangle$.

We assume that neither of the two functions $f_{l}, f_{u}$ is the identity function. The following analysis, taken from [ASY01], allows us to calculate the behaviour of cycles provided that the path along the cycle has a normal (not inverted) TAMF. Since, in good SPDIs, the TAMF between a pair of edges is normal, and the composition of two normal TAMFs is itself a normal TAMF, this approach is universally applicable as long as the goodness assumption holds.

Let $\sigma$ be a simple cycle, and $l^{*}$ and $u^{*}$ be the fix-points ${ }^{2}$ of $f_{l}$ and $f_{u}$, respectively, and $S_{\sigma} \cap J_{\sigma}=\langle L, U\rangle$. It can be shown that $\sigma$ is of one of the following types: STAY: The cycle is not abandoned neither by the leftmost nor the rightmost trajectory, that is, $L \leq l^{*} \leq u^{*} \leq U$. DIE: The rightmost trajectory exits the cycle through the left (consequently the leftmost one also exits) or the leftmost trajectory exits the cycle through the right (consequently the rightmost one also exits), that is, $u^{*}<L \vee l^{*}>U$. EXIT-BOTH: The leftmost trajectory exits the cycle through the left and the rightmost one through the right, that is, $l^{*}<L \wedge u^{*}>U$. EXIT-LEFT: The leftmost trajectory exits the cycle (through the left) but the rightmost one stays inside, that is, $l^{*}<L \leq$ $u^{*} \leq U$. EXIT-RIGHT: The rightmost trajectory exits the cycle (through the right) but the leftmost one stays inside, that is, $L \leq l^{*} \leq U<u^{*}$.

The classification above provides useful information about the qualitative behaviour of trajectories. Any trajectory that enters a cycle of type DIE will eventually leave it after a

\footnotetext{
${ }^{2}$ The fix-point $x^{*}$ is the solution of $f\left(x^{*}\right)=x^{*}$, where $f(\cdot)$ is positive affine. The existence and computation of such fix-points are detailed in [ASY07].
} 


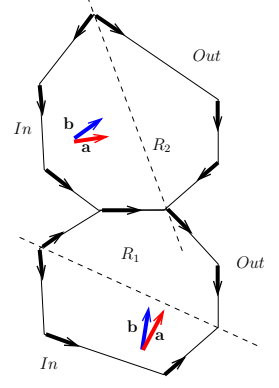

(a)

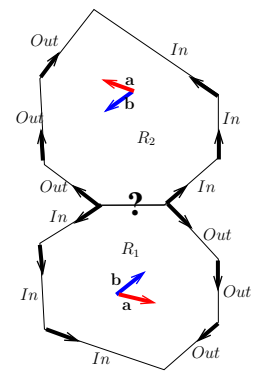

(b)

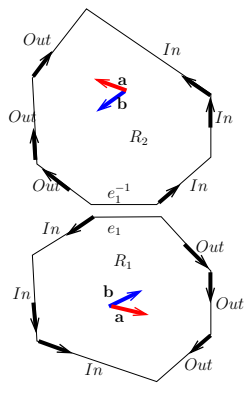

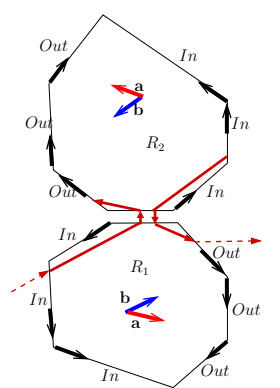

(d)

Fig. 3. (a) An SPDI with matching order of edges; (b) a GSPDI showing that the order breaks the contiguity of the edge directions; (c) a GSPDI with a duplicated inout edge; (d) a path through the GSPDI using edge $e_{1}$ in both directions.

finite number of turns. In the case of a cycle is of type STAY, all trajectories that happen to enter it will keep turning inside it forever. In all other cases, some trajectories will turn for a while and then exit, and others will continue turning forever. This information is crucial for solving the reachability problem for SPDIs. Also note that the above analysis gives us a non-iterative solution of cycle behaviour for most cycles. An important result to prove the decidability of SPDIs is that any valid signature can be expressed in a normal form, consisting of alternating sequential paths and simple loops:

Theorem 2. Given an SPDI with the goodness constraint, any edge signature $\sigma=$ $e_{1} \ldots e_{p}$ can be written as $\sigma_{\mathcal{A}}=r_{1} s_{1}^{k_{1}} \ldots r_{n} s_{n}^{k_{n}} r_{n+1}$, where for any $1 \leq i \leq n+1, r_{i}$ is a sequence of pairwise different edges and for all $1 \leq i \leq n, s_{i}$ is a simple cycle (no edges are repeated within $s_{i}$ ).

This representation of signatures is the base to obtain types of signatures with the following properties:

Lemma 2. Given a good SPDI, let $\sigma=e_{0} \ldots e_{p}$ be a feasible signature, then its type, type $(\sigma)=r_{1}, s_{1}, \ldots, r_{n}, s_{n}, r_{n+1}$ satisfies the following properties: (i) every $1 \leq i<$ $j \leq n+1, r_{i}$ and $r_{j}$ are disjoint; (ii) every $1 \leq i<j \leq n, s_{i}$ and $s_{j}$ are different.

The finiteness of the different types of signatures is the basis of the proof of decidability of (good) SPDI reachability, and of the termination of the reachability algorithm (together with acceleration results for simple loops).

Theorem 3. The reachability problem for SPDIs (satisfying goodness) is decidable.

\section{Relaxing Goodness: Generalised SPDIs}

The original proof of the decidability of the reachability question for SPDIs, depended on the concept of monotonicity of TAMFs and their composition. Before starting the analysis, the algorithm fixed the direction of the edges separating regions. An interesting 
result guaranteed that the orientation of the edges resulted in each polygon split into two contiguous sequences of paths - one being the input edges, the other being the output edges. Furthermore, the orientation of an edge in one region is guaranteed to match the orientation of the same edge in the adjacent region ${ }^{3}$, as shown in Fig. 3-(a). When one moves on to GSPDIs, inout edges (those that may be traversed in both directions) break this result, since the direction of an edge when considered as an input edge clashes with the direction it is given when used as an output edge in the same region. The previous result however, still guaranteed that the entry-only edges and the exit-only edges can be assigned in one fixed direction (see Fig. 3-(b)).

To solve this problem, we use directed edges, and differentiate between the edge used as an input, and when it is used as an output, just as though they were two different edges in the GSPDI. Fig. 3-(c) shows how an inout edge can be seen in this manner. Note that edge $e_{1}$ is an input edge in region $R_{1}$, but an output edge in region $R_{2}$, and similarly, $e_{1}^{-1}$ is an output edge in region $R_{1}$ and an input edge in region $R_{2}$. In other words, any path passing through the edge such as $\sigma=e_{0} e_{1} e_{2} \ldots e_{3} e_{1}^{-1} e_{4}$ (see Fig. 3-(d)) can be analysed as before, and through monotonicity, one can deduce that Succ $_{\sigma}$ is a positive TAMF. $e_{1}$ and $e_{1}^{-1}$ are considered distinct edges, and the above path contains no loop. It can be seen that the standard analysis for SPDIs works well for such cases. However, paths can now 'bounce' off an edge. Recall that any pair of edges $e_{0} e_{1}$ is part of a path if $e_{0}$ is an input edge of a region, and $e_{1}$ is an output edge of the same region. One can calculate the TAMF for such a trajectory. However, $e e^{-1}$ can now be a valid path, whose behaviour cannot be expressed as a normal TAMF. This breaks the analysis used in SPDIs, to accelerate the analysis of loops. The standard SPDI analysis thus needs to be extended to handle such 'bounces' in paths.

\subsection{Preliminary Results}

The goodness restriction was originally introduced to simplify treatment of trajectories and to guarantee that each region can be partitioned into entry-only and exit-only edges in an ordered way, a fact used in the proof of decidability of the reachability problem. In this section, we will introduce further background, and provide new results concerning GSPDIs, needed to prove our decidability result.

Definition 3. An edge $e \in P$ is an inout edge of $P$ if $e$ is neither an entry-only nor an exit-only edge of $P$.

An SPDI without the goodness restriction is called a Generalised SPDI (GSPDI). Thus, in GSPDIs there are three kinds of edges: inouts, entry-only and exit-only.

Self-crossing of trajectory segments of SPDIs can be eliminated which allow us to consider only non-crossing trajectory (segments). Lemma 1 (the full proof of which can be found in [ASY07]) also applies to GSPDIs. Therefore, in what follows, we will consider only trajectory segments without self-crossings. Note that on GSPDIs, a trajectory can "intersect" an edge at an infinite number of points by sliding along it. A trace is thus no longer a sequence of points, but rather, a sequence of intervals.

\footnotetext{
${ }^{3}$ There are special cases when an edge is an entry-only to a region and an exit-only to an adjacent region. From the reachability point of view this does not cause any problem as these cases can be identified and treated accordingly.
} 
Definition 4. The trace of a trajectory $\xi$ is the sequence trace $(\xi)=I_{0} I_{1} \ldots I_{n}$ of the intersection intervals of $\xi$ with the set of edges: $I_{i} \subseteq \xi \cap E_{d}(\mathcal{H})$.

Definition 5. An edge signature (or simply a signature) of a GSPDI is a sequence of edges. The edge signature of a trajectory $\xi \operatorname{Sig}(\xi)$, is the ordered sequence of traversed edges by the trajectory segment, that is, $\operatorname{Sig}(\xi)=e_{0} e_{1} \ldots e_{n}$, with $\operatorname{trace}(\xi)=$ $I_{0} I_{1} \ldots I_{n}$ and $I_{i} \subseteq e_{i}$.

Note that, in many cases, the intervals of a trace are in fact points. We say that a trajectory with edge signature $\operatorname{Sig}(\xi)=e_{0} e_{1} \ldots e_{n}$ and trace $\operatorname{trace}(\xi)=I_{0} I_{1} \ldots I_{n}$ interval-crosses edge $e_{i}$ if $I_{i}$ is not a point. Given a trajectory segment, we will distinguish between proper inout edges and sliding edges.

Definition 6. Let $\xi$ be a trajectory segment from point $\mathbf{x}_{0} \in e_{0}$ to $\mathbf{x}_{f} \in e_{f}$, with edge signature $\operatorname{Sig}(\xi)=e_{0} \ldots e_{i} \ldots e_{n}$, and $e_{i} \in E(P)$ be an edge of $P$. We say that $e_{i}$ is a sliding edge of $P$ for $\xi$ if $\xi$ interval-crosses $e_{i}$, otherwise e is said to be a proper inout edge of $P$ for $\xi$.

We say that a trajectory segment $\xi$ slides along an edge $e$, if $e$ is a sliding edge of $P$ for $\xi$, and that $\xi$ is a sliding trajectory if it contains at least one sliding edge.

The signatures that we will be analysing in GSPDIs are similar to ones in SPDIs, except that they may include inverted edges of the form $e$ and $e^{-1}$. The behaviour between such edges does not correspond to a normal TAMF, and thus has to be analysed separately. One interesting property of inout edges is that the dynamics of the region they are in allow us to slide along the edge to one of the end-points of the edge.

Proposition 1. If $e$ is an inout edge, then any trajectory reaching the edge can always slide on the edge (in one or the other direction, or both).

As for SPDIs, we have the following property of Succ: for any edge signatures $\sigma_{1}$ and $\sigma_{2}$ and edge $e$ : Succ $_{e \sigma_{1}} \circ$ Succ $_{\sigma_{2} e}=$ Succ $_{\sigma_{2} e \sigma_{1}}$.

The following lemma shows that the edge-to-edge successor function is a normal TAMF whenever the two edges are not the inverse of each other. It follows directly from the similar result for SPDIs [ASY07], which makes no assumption regarding goodness.

Lemma 3. For any two edges $e_{0}$ and $e_{1}$, Succ $_{e_{0} e_{1}}$ is always a normal TAMF, whenever $e_{1} \neq e_{0}^{-1}$.

A bounce is a part of a trajectory which crosses an edge twice in immediate succession. We define bounces formally within a signature as follows:

Definition 7. Given a signature $\sigma=e_{0} e_{1} \ldots e_{n}$, a pair of edges $e_{i} e_{i+1}$ is said to be a bounce if $e_{i+1}=e_{i}^{-1}$. We say that a signature $e_{0} e_{1} \ldots e_{n}$ contains $m$ bounces, if there are exactly $m$ distinct indices $I=\left\{i_{1}, i_{2}, \ldots i_{m}\right\}$ such for every $i \in I, e_{i}=e_{i+1}^{-1}$.

Let Flip $[l, u]=[1-u, 1-l]$ be an interval function. The following result establishes that the successor function for bounces can be defined in terms of the Flip function. The result follows directly from the definition of $e^{-1}$ : 
Lemma 4. The behaviour of going from an edge e to its inverse $e^{-1}$ is equivalent to Flip. In other words: Succ $_{e e^{-1}}=$ Flip.

One of the useful properties of SPDIs is that the successor function of any given signature is a normal TAMF. For GSPDIs, however, we need to take into account bounces, and hence analyse the composition of normal TAMFs with Flip:

Lemma 5. Composing Flip with an inverted TAMF gives a normal TAMF and an inverted TAMF if we compose it with a normal TAMF.

The parity of the number of bounces occurring in a given signature influences the form of the underlying TAMF, as shown in the following result, whose proof follows immediately by induction on the number of bounces.

Corollary 2. Any signature with an even number of bounces has its behaviour characterised by a normal TAMF, while a signature with an odd number of bounces is characterised by an inverted TAMF.

Given a simple cycle $\sigma$, let $\sigma^{+}$be the cycle iterated one or more times. Recall that the analysis of simple cycle behaviour given for SPDIs depended only on the assumption that the TAMF of the cycle body is a normal one. From the previous result, it thus follows that whenever the number of bounces is even on a given cyclic signature, the composed TAMF is a normal one, so the loop analysis can be conducted as for SPDIs:

Lemma 6. Given a loop $\sigma$ containing an even number of bounces, its iterated behaviour $\sigma^{+}$can be calculated as for SPDIs.

Since we slide along inout edges, and can only bounce off inout edges, we can prove that loops which include at least one bounce are never STAY loops:

Lemma 7. Loops which include bounces are not STAY loops.

This leaves only simple cycles with an odd number of bounces to be analysed. Considering the case when a bounce appears as the first pair of elements of a loop body, we can accelerate the analysis by running through the loop only once. The proof follows from the fact that the initial bounce enables a slide, thus allowing us to identify the limits through only one application of the loop body:

Lemma 8. Given a signature $\sigma=e_{0}\left(e_{1} e_{1}^{-1} e_{2} \ldots e_{n}\right)^{k} e_{1}$ (i) with only one loop; (ii) with $k>0$; (iii) which has an odd number of bounces; and (iv) starts with a bounce; the behaviour of signature is equivalent to following the loop only once as in $\sigma^{\prime}=$ $e_{0} e_{1} e_{1}^{-1} e_{2} \ldots e_{n} e_{1}$. In other words: Succ $_{\sigma}=$ Succ $_{\sigma^{\prime}}$.

Based on the above lemma, we can prove that any loop containing an odd number of bounces can be accelerated. The proof works by unwinding the loop body to push the first bounce to the beginning, and then applying the previous lemma:

Lemma 9. Given a loop $s$ with an odd number of bounces, we can calculate the limit of $s^{+}$without iterating.

Therefore, we can now analyse any type of signature in GSPDIs using the results from lemma 3 (to deal with inout edges), and lemmas 6 and 9 (to deal with bounces).

Theorem 4. We can compute the behaviour of a signature $r_{1} s_{1}^{+} r_{2} s_{2}^{+} \ldots r_{n}$. 


\subsection{Decidability}

The following lemma guarantees that it is sufficient to consider simple cycles which occur in a type of signature only under certain patterns. Any type of signature containing two occurrences of the same simple cycle can be reduced to another type of signature where the simple cycle $s$ occurs only once, provided the cycle with the edges in reverse order (denoted reverse $(s)$ ) does not occur between them. The proof is based on the fact that, assuming the path does not cross itself, between two instances of a repeated loop, one can always find either (i) the reverse of the cycle; or (ii) a bounce. In the latter case, it can be shown that the bounce can be eliminated to avoid leaving the loop.

Lemma 10. Given a GSPDI, and assuming only trajectories without self-crossing, if there is a type of signature where a simple cycle $s=\left(e_{0}, e_{1}, \ldots, e_{n}\right)$ appears twice, i.e. type $(\operatorname{Sig}(\xi))=\sigma^{\prime} \sigma^{\prime \prime} \sigma^{\prime \prime \prime}$ with $\sigma^{\prime \prime}=s^{k} \ldots s^{k^{\prime \prime}}$, then if there is no reverse $(s)$ between the two occurrences of $s$, then type $(\operatorname{Sig}(\xi))=\sigma^{\prime} s^{k^{\prime \prime \prime}} \sigma^{\prime \prime \prime}$.

We also prove that a trajectory which takes a loop (any number of times), then takes it again (once again any number of times) but in reverse order, and finally takes it a number of times in the forward direction, can be simulated by another trajectory which simply takes the loop a number of times. The proof is based on the fact that whichever direction the first edge of the simple cycle under consideration allows sliding in, it is possible to obtain a type of signature preserving reachability without such a pattern.

Lemma 11. Given a GSPDI, if there is a trajectory segment $\xi:[0, T] \rightarrow \mathbb{R}^{2}$, with $\xi(0)=\mathbf{x}$ and $\xi(t)=\mathbf{x}^{\prime}$ for some $t>0$, such that type $(\operatorname{Sig}(\xi))=r_{1} s_{1}^{k_{1}} r_{2} s_{2}^{k_{2}} r_{3} s_{3}^{k_{3}} r_{4}$, with $s_{2}=s_{1}^{-1}$ and $s_{3}=s_{1}$, then it is always possible to find a trajectory segment $\xi^{\prime}$ : $[0, T] \rightarrow \mathbb{R}^{2}$ such that $\xi^{\prime}(0)=\mathbf{x}$ and $\xi^{\prime}(t)=\mathbf{x}^{\prime}$ for some $t>0$, and type $(\operatorname{Sig}(\xi))=$ $r_{1} s_{1}^{k_{1}^{\prime}} r_{4}^{\prime}$.

Based on the above, we can conclude that for GSPDIs we can always transform a type of signature into one where simple loops are not repeated.

Corollary 3. Given a GSPDI, let $\sigma$ be an edge signature, then it can always be written as $\sigma_{\mathcal{A}}=r_{1} s_{1}^{k_{1}} \ldots r_{n} s_{n}^{k_{n}} r_{n+1}$, where for any $1 \leq i \leq n+1, s_{i}$ is a simple cycle (i.e., without repetition of edges), and for every $1 \leq i<j \leq n, s_{i}$ and $s_{j}$ are different.

The following lemma, ensuring that there are a finite number of types of signatures in GSPDIs, follows from the previous results and it is the basis for the termination proof of the reachability algorithm.

Corollary 4. For a GSPDI there are finitely many different types of signatures.

\subsection{Algorithm}

Given a type of signature $\sigma$ where each edge is traversed in exactly one direction, let $\operatorname{Reach}_{\sigma}\left(\mathbf{x}_{0}, \mathbf{x}_{f}\right)$ be the SPDI reachability algorithm presented in [ASY07]. The reachability algorithm for a GSPDI $\mathcal{H}, \operatorname{Re} \operatorname{each}\left(\mathcal{H}, \mathbf{x}_{0}, \mathbf{x}_{f}\right)$, consists of the following steps: (1) Generate the finite set of types of signatures $\Sigma=\left\{\sigma_{0}, \ldots, \sigma_{n}\right\}$ taking into account $e$ 
and $e^{-1}$ as different edges, and such that the loop signatures are all distinct; (2) Apply the function $\operatorname{Reach}_{\sigma_{i}}\left(\mathbf{x}_{0}, \mathbf{x}_{f}\right)$ for each $\sigma_{i} \in \Sigma$; (3) If for at least one $\sigma_{i} \in \Sigma$, $\operatorname{Reach}_{\sigma_{i}}\left(\mathbf{x}_{0}, \mathbf{x}_{f}\right)=$ Yes, then $\operatorname{Reach}\left(\mathcal{H}, \mathbf{x}_{0}, \mathbf{x}_{f}\right)=$ Yes, otherwise the answer is No. In step 2 we apply Succ progressively on the abstract signature, using lemmas 6 and 9 to compute the successor of a loop with bounces, and the Succ function as in the case of SPDIs for the rest. Based on these results, it is possible to show termination, correctness and completeness of GSPDI reachability.

Lemma 12. $\operatorname{Reach}\left(\mathcal{H}, \mathbf{x}_{0}, \mathbf{x}_{f}\right)$ is a terminating, correct and complete algorithm calculating GSPDI reachability.

From this, the main theoretical result of our paper follows immediately:

Theorem 5. The reachability problem for GSPDIs is decidable.

\section{Conclusions}

We have proved that the reachability question for GSPDIs is decidable. The proof is constructive, giving an algorithm which extends the one given in [ASY07] for SPDIs. The key lies in showing that the previous analysis works in all cases except when a loop contains an odd number of bounces. The algorithm is extended to deal with such cases. The algorithm needs to be extended to deal with inout edges which enable sliding, but the overall effect is to accelerate the analysis of an SPDI, since at least one end of the edge is immediately covered once the edge is reached.

The main contribution of our paper is interesting in a theoretical sense since it extends the class of decidable hybrid systems, narrowing further the gap between what is known to be be decidable and what is known to be undecidable [AS02,MP05]. The result is also interesting in a practical sense, since it provides a good foundation to approximate planar non-linear differential equations. We plan to implement the algorithm, extending the SPeeDI ${ }^{+}$toolkit $[\mathrm{Spe}]$ to treat GSPDIs, and use on case studies with non-linear differential equations.

\section{References}

[AS02] E. Asarin and G. Schneider. Widening the boundary between decidable and undecidable hybrid systems. In CONCUR'02, LNCS 2421. Springer-Verlag, 2002.

[ASY01] E. Asarin, G. Schneider, and S. Yovine. On the decidability of the reachability problem for planar differential inclusions. In HSCC'01, LNCS 2034. Springer-Verlag, 2001.

[ASY07] E. Asarin, G. Schneider, and S. Yovine. Algorithmic Analysis of Polygonal Hybrid Systems. Part I: Reachability. TCS, 379(1-2):231-265, 2007.

[HS74] M.W. Hirsch and S. Smale. Differential Equations, Dynamical Systems and Linear Algebra. Academic Press Inc., 1974.

[MP93] O. Maler and A. Pnueli. Reachability analysis of planar multi-linear systems. In $C A V$, LNCS 687. Springer-Verlag, 1993.

[MP05] V. Mysore and A. Pnueli. Refining the undecidability frontier of hybrid automata. In FSTTCS, LNCS 3821. Springer-Verlag, 2005.

[Sch02] G. Schneider. Algorithmic Analysis of Polygonal Hybrid Systems. PhD thesis, VERIMAG - UJF, Grenoble, France, July 2002.

[Spe] SpeeDI ${ }^{+}$. http://www.cs.um.edu.mt/speedi/. 


\section{A Proofs of Lemmas, Theorems and Propositions}

This appendix is for reviewing purposes only. It contains the detailed proofs of the results which could not be included for space reasons is being included at the end of the paper. Should the paper be accepted for publication, the main paper merged with the proofs will be published as a technical report for reference.

\section{Section 3}

Corollary 1 Composition of two TMAFs gives a TMAF. The composition of two inverted TAMFs gives a normal TAMF. Conversely, the composition of one normal and one inverted TAMF (in either order) gives an inverted TAMF.

Proof Sketch. The proof follows similarly to Theorem 1, where we note that the resulting affine functions are switched when one of the TAMFs is inverted, and the gradient is the product of the original two gradient values (hence positive when both positive or negative, and negative otherwise).

\section{Section 4.1}

Proposition 1 If $e$ is an inout edge, then any trajectory reaching the edge can always slide on the edge (in one or the other direction, or both).

Proof. The results follows from the fact that the director vector of $e$ can be expressed as the positive linear combination of the two vectors of the region in consideration.

Proposition 2. If $e_{0}$ is an inout edge, then for any other edge $e_{1}$, and interval $I$, such that $\operatorname{Succ}_{e_{1} e_{0}}(I)$ is not empty, all such applied successors include the left or all include the right end of the edge (equal to one of $(0, x\rangle$ or $\langle x, 1)$ for some value of $x-$ depending on one of I's extremities).

Proof. This is a direct consequence of Proposition 1.

Lemma 5 Composition of the function Flip with an inverted TAMF results in a normal TAMF and in an inverted TAMF if we compose Flip with a normal TAMF.

Proof. Consider a normal TAMF $f$ : 


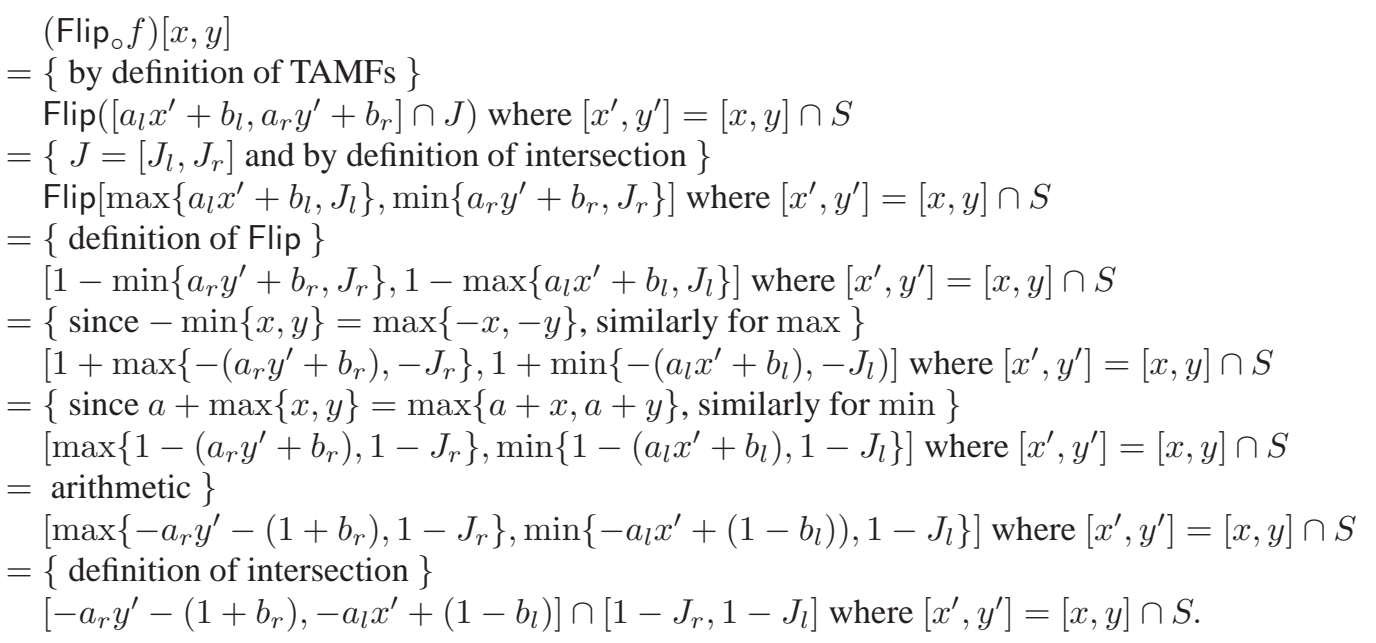

Note that the result is also an inverted TAMF. The other result follows identically.

Corollary 2 Any signature with an even number of bounces has its behaviour characterised by a normal TAMF, while a signature with an odd number of bounces is characterised by an inverted TAMF.

Proof. The proof follows by induction on the number of edges appearing in the signature.

The base case is when the signature consists of exactly two edges (shorter sequences of edges are not signatures by definition). Let the signature be $\sigma=e_{0} e_{1}$. Now either (i) $e_{1}=e_{0}^{-1}$, in which case we have an odd number (exactly one) bounce, and Succ $\sigma=$ Flip (by definition 7) which is an inverted TAMF (by definition of Flip); or (ii) $e_{1} \neq e_{0}^{-1}$, in which case we have an odd number of bounces (zero) and Succ $\sigma$ is a normal TAMF by the result in [ASY07]. In both cases, the result holds.

Now let us assume that the result holds for signatures of length $n$, and we will consider a signature of length $n+1$, namely: $\sigma=e_{0} e_{1} \ldots e_{n}$. Once again, either $e_{n}=e_{n-1}^{-1}$ or it is not. We will consider the cases separately:

- If $e_{n}=e_{n-1}^{-1}$, then the signature $e_{0} e_{1} \ldots e_{n-1}$ contains one bounce less that the original signature.

$$
\begin{aligned}
& \text { Succ }_{e_{0} e_{1} \ldots e_{n}} \\
= & \{\text { property of Succ }\} \text { Succe }_{n-1} e_{n} \circ \text { Succ }_{e_{0} e_{1} \ldots e_{n-1}} \\
= & \{\text { definition of Succ on a bounce }\} \\
& \text { Flip } \circ \text { Succ }_{e_{0} e_{1} \ldots e_{n-1}}
\end{aligned}
$$

Now, if $e_{0} e_{1} \ldots e_{n}$ has an even number of bounces, $e_{0} e_{1} \ldots e_{n-1}$ has an odd number of bounces (since the last pair were a bounce), and thus, by the inductive hypothesis, Succ $_{e_{0} e_{1} \ldots e_{n-1}}$ is an inverted TAMF. But by the above equational reasoning, and lemma 5, it follows that Succ $_{e_{0} e_{1} \ldots e_{n}}$ is a normal TAMF.

The case when $e_{0} e_{1} \ldots e_{n}$ has an odd number of bounces follows similarly. 
- On the other hand, if $e_{n} \neq e_{n-1}^{-1}$, then the signature $e_{0} e_{1} \ldots e_{n-1}$ contains the same number of bounces as the original signature.

$$
\begin{aligned}
& \text { Succ }_{e_{0} e_{1} \ldots e_{n}} \\
= & \{\text { property of Succ }\} \\
& \text { Succe }_{n-1} e_{n} \circ \text { Succ }_{e_{0} e_{1} \ldots e_{n-1}}
\end{aligned}
$$

As before, if $e_{0} e_{1} \ldots e_{n}$ contains an even number of bounces, so thus $e_{0} e_{1} \ldots e_{n-1}$ (since the last pair were not a bounce), and thus, by the inductive hypothesis, Succ $_{e_{0} e_{1} \ldots e_{n-1}}$ is a normal TAMF. But by the above equational reasoning, and lemma 1, it follows that Succ $_{e_{0} e_{1} \ldots e_{n}}$ is a normal TAMF.

The case when $e_{0} e_{1} \ldots e_{n}$ has an odd number of bounces follows similarly.

Lemma 7 Loops which include bounces are not STAY loops.

Proof. The proof follows from Proposition 1, which guarantees that once we reach the first inout edge, we can always slide to one end of the edge. Hence any loop containing such edge cannot be a STAY, by definition.

Lemma 6 The behaviour of any loop $\sigma$ containing an even number of bounces can be calculated as for SPDIs.

Proof. Corollary 2 ensures that $\mathrm{Succ}_{\sigma}$ is a normal TAMF. Earlier, in Section 2, we have summarised the analysis from [ASY01] which enables us to calculate the behaviour of a cycle whose TAMF is not inverted, in a non-iterative manner. We can thus use this technique to calculate the iterated behaviour of $\sigma$ in a non-iterative way.

Lemma 8 Given a signature with one loop $\sigma=e_{0}\left(e_{1} e_{1}^{-1} e_{2} \ldots e_{n}\right)^{k} e_{1}$ (with $k>0$, which has an odd number of bounces, and starts with a bounce), the behaviour of signature is equivalent to following the loop only once as in $\sigma^{\prime}=e_{0} e_{1} e_{1}^{-1} e_{2} \ldots e_{n} e_{1}$. In other words: Succ $_{\sigma}=$ Succ $_{\sigma^{\prime}}$.

Proof. Since $e_{1}$ is an inout edge, by proposition 2, we know that we can slide in (at least) one direction. without loss of generality, let's assume that for any $e, \operatorname{Succ}_{e e_{1}}(I)=$ $(0, x)$. Note that due to the definition of TAMFs, $x$ is only dependant on the right bound of $I$.

Let $F=\operatorname{Succ}_{e_{1}^{-1} e_{2} \ldots e_{n} e_{1}}$. Since this includes an even number of bounces, composed TAMF (thus $F$ ) is a normal (non-inverted) TAMF. Moreover, since $F(I)=\operatorname{Succ}_{e_{n} e_{1}}\left(\operatorname{Succ}_{e_{1}^{-1} e_{2} \ldots e_{n}}(I)\right.$ ), then $F(I)=(0, x)$ for some value of $x$. Finally, we note that since $F$ is a normal TAMF, $x$ is dependant only on the right bound of $I$, we can conclude that there exists $\alpha$ such that for any $x, F(x, 1)=(0, \alpha)$.

We can now proceed to prove the result by induction on $k$. Trivially, the result holds for $k=1$. Now consider $k>1$ : 


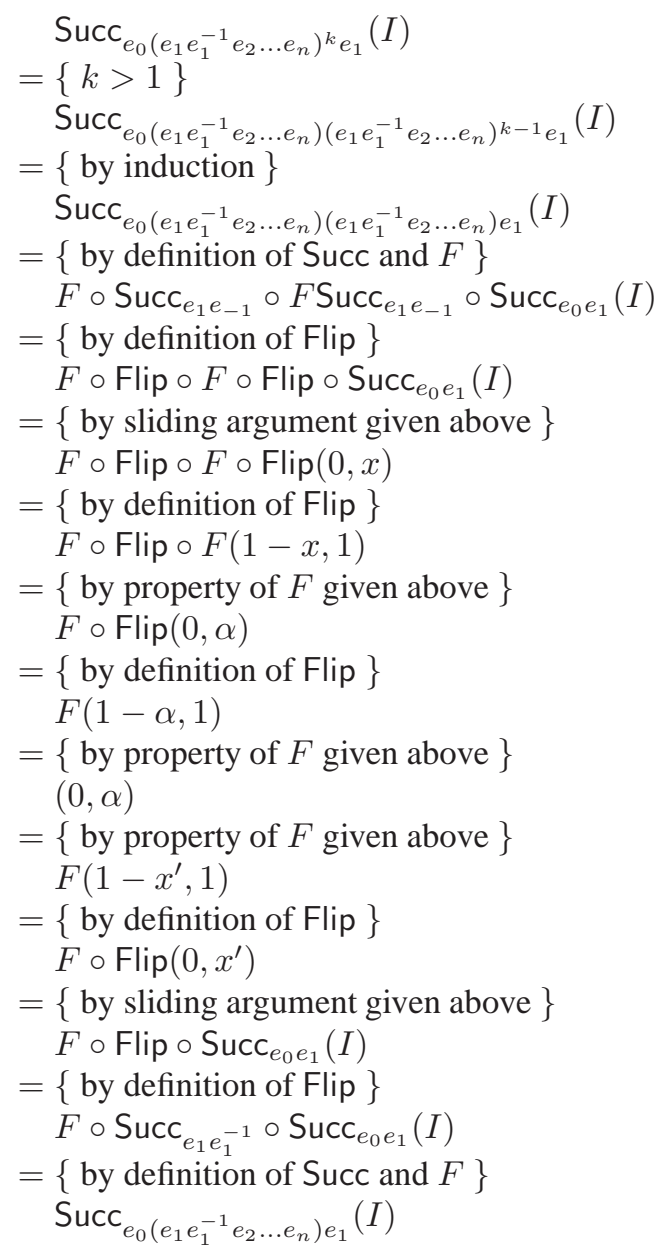

By induction the result thus follows.

Lemma 9 Given a loop $\sigma$ with an odd number of bounces, we can calculate the limit of $\sigma^{+}$without iterating.

Proof. Let $\sigma=e_{0} e_{1} \ldots e_{i} e_{i}^{-1} e_{i+1} \ldots e_{n}$, where $e_{i} e_{i}^{-1}$ is the first bounce of the sequence. Since $\sigma$ contains inout edges, it cannot be a STAY loop, and we only consider the case where the loop finally exits. Consider the exiting loop $\sigma^{+} e^{\prime}$.

The case when $\sigma$ is never repeated or repeated only once, can be easily handled. When the number of repetitions is at least twice, we can use the following reasoning: 


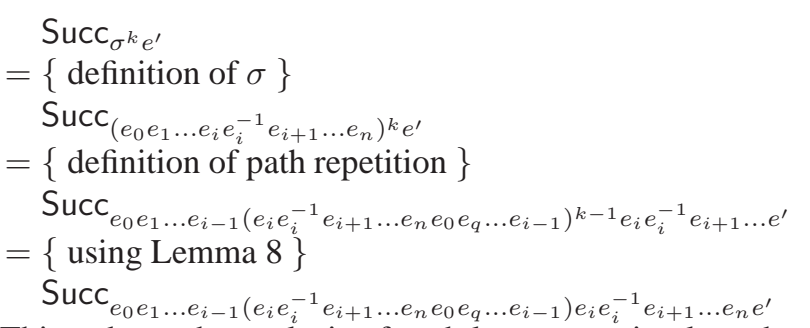

This reduces the analysis of such loops to a simple path analysis which we know how to perform.

Theorem 4 We can (constructively) compute the behaviour of a signature $r_{1} s_{1}^{+} r_{2} s_{2}^{+} \ldots r_{n}$.

Proof. We use the standard techniques presented in [ASY07], but use Theorems 6 and 9 to analyse loops with bounces.

\section{Section 4.2}

Lemma 10 Given a GSPDI, and assuming only trajectories without self-crossing, if there is a type of signature where a simple cycle $s=\left(e_{0}, e_{1}, \ldots, e_{n}\right)$ appears twice, i.e. type $(\operatorname{Sig}(\xi))=\sigma^{\prime} \sigma^{\prime \prime} \sigma^{\prime \prime \prime}$ with $\sigma^{\prime \prime}=s^{k} \ldots s^{k^{\prime \prime}}$, then if there is no reverse $(s)$ between the two occurrences of $s$, then type $(\operatorname{Sig}(\xi))=\sigma^{\prime} s^{k^{\prime \prime \prime}} \sigma^{\prime \prime \prime}$.

Proof Sketch. There are two cases:

1. $\sigma^{\prime \prime}=s^{k} r s^{k^{\prime \prime}}$ : In this case $r$ must be of the form $e_{n}^{-1} e_{n-1}^{-1} \ldots e_{i}^{-1}$ with $i>0$. We must have a bouncing at $e_{i}^{-1}$, then we can slide and we get $\sigma^{\prime \prime}=s^{k^{\prime \prime \prime}}$.

2. $\sigma^{\prime \prime}=s^{k} \omega s^{k^{\prime \prime}}$ : Here $\omega$ is any finite sequence of alternating $r$ 's and $s^{\prime}$ s. It can be shown that either we reduce to the previous case, or $\omega$ must contain reverse $(s)$, or there must be a self-crossing.

Lemma 11 Given a GSPDI, if there is a trajectory segment $\xi:[0, T] \rightarrow \mathbb{R}^{2}$, with $\xi(0)=\mathbf{x}$ and $\xi(t)=\mathbf{x}^{\prime}$ for some $t>0$, such that type $(\operatorname{Sig}(\xi))=r_{1} s_{1}^{k_{1}} r_{2} s_{2}^{k_{2}} r_{3} s_{3}^{k_{3}} r_{4}$, with $s_{2}=\operatorname{reverse}\left(s_{1}\right)$ and $s_{3}=s_{1}$, then it is always possible to find a trajectory segment $\xi^{\prime}:[0, T] \rightarrow \mathbb{R}^{2}$ such that $\xi^{\prime}(0)=\mathbf{x}$ and $\xi^{\prime}(t)=\mathbf{x}^{\prime}$ for some $t>0$, and $\operatorname{type}(\operatorname{Sig}(\xi))=r_{1} s_{1}^{k_{1}^{\prime}} r_{4}^{\prime}$.

Proof Sketch. Let $s_{1}=\left(e_{0}, e_{1}, \ldots, e_{n}\right)$ be a simple cycle where $\xi$ is a clockwise spiral turning inwards. Due to Proposition 1, we have the following two cases:

1. $e_{0}$ allows sliding inwards. We can always eliminate the first $s$, i.e., $\operatorname{type}(\operatorname{Sig}(\xi))=$ $r_{1}^{\prime} s_{2}^{k_{2}} r_{3} s_{3}^{k_{3}} r_{4}$. See Fig. 6 .

2. $e_{0}$ allows sliding outwards. Two cases:

(a) reverse $\left(s_{1}\right)$ loops outwards. In this case we can eliminate $s_{2}$ since once we start that loop, we can slide outwards till $s_{3}$ starts, and we get type $(\operatorname{Sig}(\xi))=$ $r_{1} s_{1}^{k_{1}} s_{3}^{k_{3}} r_{4}$, which is type $(\operatorname{Sig}(\xi))=r_{1} s_{1}^{k^{\prime}} r_{4}$. See Fig. 4-(a).

(b) reverse $\left(s_{1}\right)$ loops inwards. Two cases: 

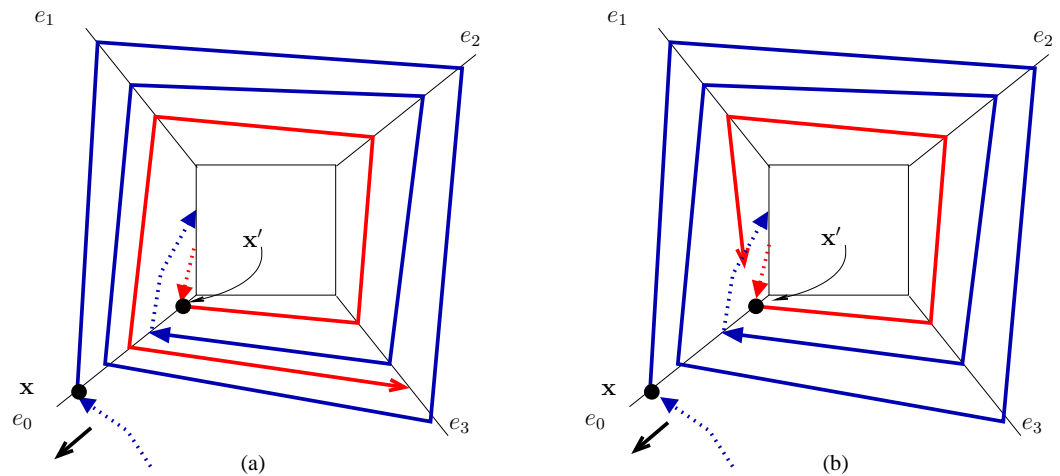

Fig. 4. Proof of Lemma 11 - Case sliding outwards: (a) case reverse $(s)$ looping outwards; (b) case reverse $(s)$ looping inwards and exiting.
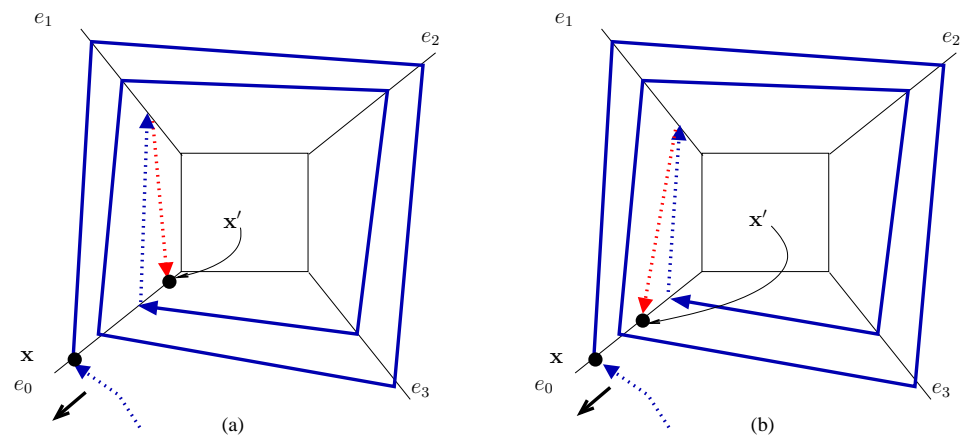

Fig. 5. Proof of Lemma 11 - Case sliding outwards: (a) case bouncing inwards; (b) case bouncing outwards.

i. $r_{2}$ contains only edges in $s_{1}$ and $s_{2}$. This implies bouncing. Two cases.

A. Bouncing inwards. Implies sliding inwards, which contradicts the assumption. See Fig. 5-(a).

B. Bouncing outwards. Implies reverse $\left(s_{1}\right)$ must loop outwards, contradicts the assumption that reverse $\left(s_{1}\right)$ loops inwards. See Fig. 5-(b).

ii. $r_{2}$ contains edges not in $s_{1}$ and $s_{2}$. This means that the trajectory exit $s_{1}$ through the 'right'. Let us assume the last visited point in $s_{1}$ is $\mathbf{x} \in e_{n}$, and that $\mathbf{x}^{\prime} \in e$ such that $\xi(t)=\mathbf{x}$ and $\xi\left(t^{\prime}\right)=\mathbf{x}^{\prime}$ with $\operatorname{Sig}\left(\xi\left[t . t^{\prime}\right]\right)=e_{n} e$, where $e \in \operatorname{first}\left(r_{2}\right)$. Then the segment of line $\overline{\mathbf{x x}^{\prime}}$ partition the region $R$ into two subregions $R_{1}$ and $R_{2}$. Clearly the only way to have $r_{2} s_{2}$ with $s_{2}$ going inwards is from a trajectory segment from region $R_{1}$ to $R_{2}$ by crossing $\overline{\mathrm{xx}^{\prime}}$, which breaks the assumption of non-crossing trajectories. Thus the pattern $s_{2} r_{3} s_{3}$ is not possible in this case. See Fig. 4-(b).

Corollary 3 Given a GSPDI, let $\sigma=e_{1} \ldots e_{p}$ be an edge signature, then it can always be written as $\sigma_{\mathcal{A}}=r_{1} s_{1}^{k_{1}} \ldots r_{n} s_{n}^{k_{n}} r_{n+1}$, where for any $1 \leq i \leq n+1, s_{i}$ is a simple 


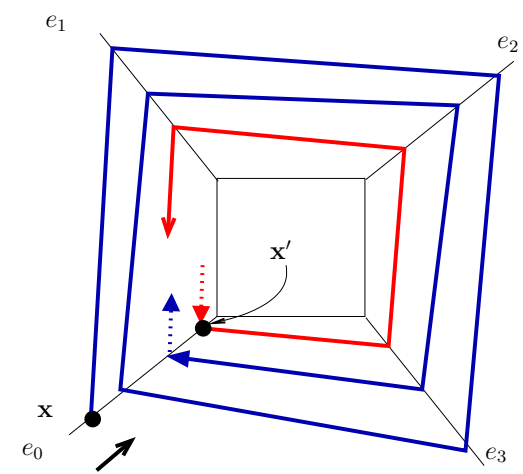

Fig. 6. Proof of Lemma 11 -Case sliding inwards.

cycle (i.e., without repetition of edges), and for every $1 \leq i \neq j \leq n, s_{i}$ and $s_{j}$ are different.

Proof. If there are $i \neq j$ such that $s_{i}=s_{j}$, the only possibility is to satisfy or the assumptions of Lemma 10 or Lemma 11. In both cases we can always obtain a signature without repeating $s_{i}$.

Corollary 4 The number of different types of abstract signatures of a given GSPDI is finite.

Proof. Based on Lemma 3, it suffices to analyse signatures of the form $\sigma_{\mathcal{A}}=r_{1} s_{1}^{+} \ldots r_{n} s_{n}^{+} r_{n+1}$ such that provided that $i \neq j, s_{i} \neq s_{j}$ and with each $r_{k}$ containing no repeated edges. Hence, since the number of edges is finite, the number of possible values each $r_{k}$ can take is finite. Similarly, the number of distinct simple loops is finite. Therefore, the number of abstract signatures to analyse is finite.

\section{Section 4.3}

Lemma $12 \operatorname{Reach}\left(\mathcal{H}, \mathbf{x}_{0}, \mathbf{x}_{f}\right)$ is a terminating, correct and complete algorithm calculating GSPDI reachability.

Proof. Termination of step 1 follows from the fact that GSPDIs have finite partitions. Step 2 terminates by corollary 4. Using Theorems 9 and 6 we can also compute steps 3 and 4 , hence guaranteeing termination of the algorithm.

Correctness of the algorithm follows from Theorems 9 and 6 (on accelerating loops with bounces) and the results in [Sch02,ASY07] on the correctness of SPDI reachability checking.

Finally, completeness is guaranteed by Theorem 4 .

Therefore, $\operatorname{Reach}\left(\mathcal{H}_{i}, \mathbf{x}_{0}, \mathbf{x}_{f}\right)$ (for all $\mathcal{H}_{i} \in \mathcal{H}_{\text {red }}, 1 \leq i \leq n$ ), is a terminating complete and sound algorithm for deciding GSPDI reachability. 